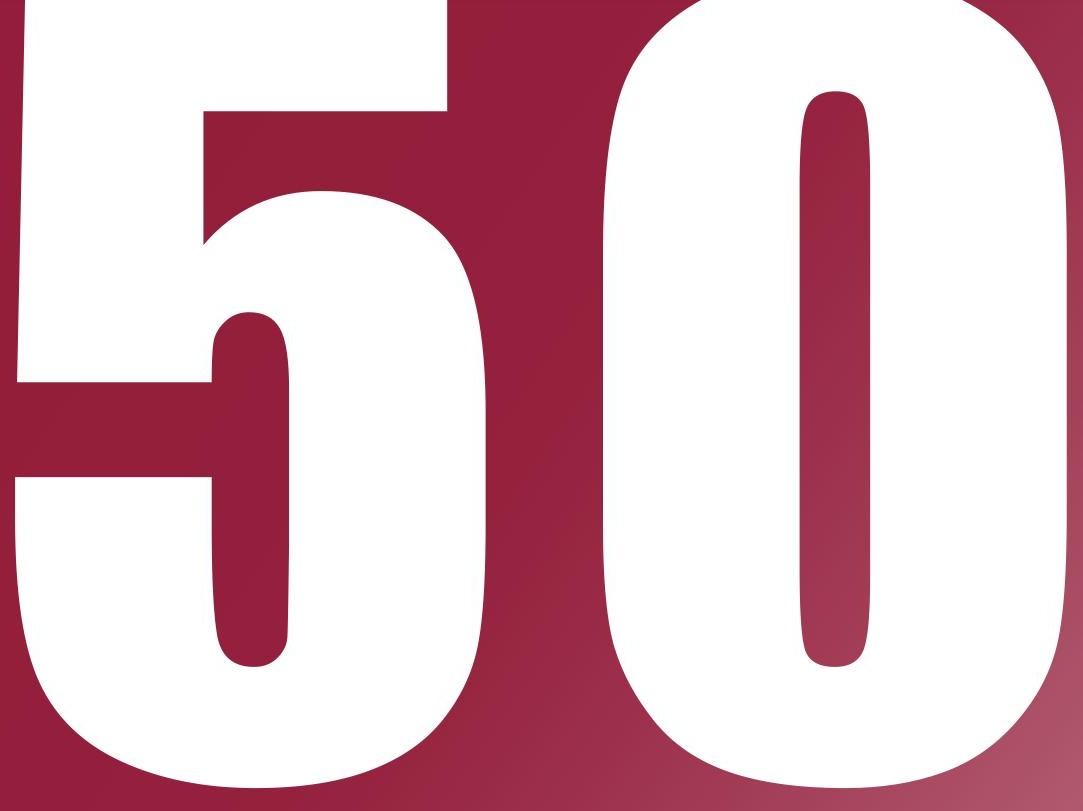

ÁMBITOS

REVISTA

INTERNACIONAL

DE

COMUNICACIÓN

$\mathbf{N}^{\circ} \mathbf{5 0}$

EDICIÓN OTOÑO

2020

ISSN: 1139-1979

E-ISSN: 1988-5733

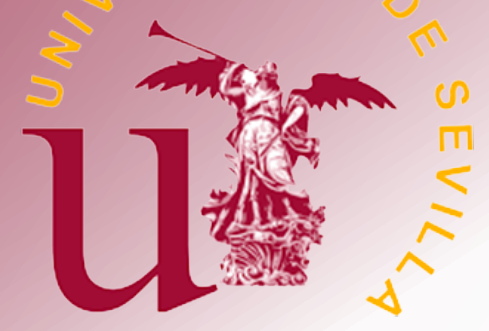




\section{INDICE}

EDITORIAL EDITORIAL

Presentación del Monográfico. Comunicación y juventud. Nuevos medios, representación, recepción y participación en contenidos de entretenimiento e información

Presentation of the monograph. Communication and youth. New media, representation, reception and participation in entertainment and information content.

Juan Francisco Gutiérrez Lozano, Francisco Javier Ruiz del Olmo

7-9

\section{MONOGRÁFICO MONOGRAPH}

El consumo audiovisual de la Generación Z. El predominio del vídeo online sobre la televisión tradicional The audiovisual consumption of Generation $Z$. The predominance of online video over traditional television

La imagen de los jóvenes en las estrategias transmedia de las series de televisión: el caso de Skam España en Instagram

The image of young people in transmedia strategies of TV series: the case of Skam España on Instagram

Sergio Jesús Villén Higueras, Francisco Javier Ruiz del Olmo

La penetración de Netflix en el público español ¿cuestiona el modelo televisivo tradicional?

Does the arrival of Netflix in the Spanish audience challenge the traditional television model?

Javier Bustos Díaz

49-61

Generación Z y consumo de información política: entre la televisión y los nuevos formatos mediáticos Generation $Z$ and consumption of political information: between television and new media formats

Ruth Gómez de Travesedo Rojas, Marta Gil Ramírez

$62-79$

Actualidad y comedia: El éxito de El Intermedio y La Resistencia entre los espectadores jóvenes

Information and comedy: The Success of El Intermedio and La Resistencia for young audiences

Inmaculada Concepción Aguilera García

80-95 
El efecto socializador de articular un espacio de comunidad virtual en el ecosistema del aula

The socializing effect of creating a virtual community space in the classroom environment

Lorea Ariadna Ruiz Gómez, Mónica Hinojosa Becerra, Francisco Javier Ruiz San Miguel

Juventude ciberativista e educação: reflexões sobre um jeito hacker de ser

Cyber activity youth and Education: reflections on a hacker way of being

Carla Azevedo de Aragão, Pietro Matheus Bompet Fontoura Alves, Karina Moreira Menezes

La risa grotesca en la obra de Bob Dylan: análisis de la Bacanal y el Festín Carnavalesco en la trilogía folk-rock eléctrica

Grotesque Laughter in Bob Dylan's Work: Analysis of The Bacchanal and the Carnival Feast in the Electric Folk-rock Trilogy

Jesús Albarrán Ligero

El papel del intertexto en el videojuego. Una partida, mil y una historias

Intertext in video games. A game, thousand and one stories

Rocío Serna-Rodrigo

145-158

\section{ÁmBitos PERSONALES PERSONAL ÁmBITOS}

El auge de Twitch: nuevas ofertas audiovisuales y cambios del consumo televisivo entre la audiencia juvenil The rise of Twitch: New audiovisual offers and the transformation of Television consumption among young audiences

\section{ARTÍCULOS ARTICLES}

La recepción de los medios de comunicación para residentes rusos en la Costa del Sol

Mass media reception by Russian residents on the Costa del Sol

Natalia Meléndez Malavé, José Carlos Pozo García

176-192

La calidad periodística en la cobertura de terremotos: Caso Ecuador

Journalistic quality in earthquake coverage: The case of Ecuador

Juan Pablo Arrobo-Agila, Mendoza María, Ignacio Aguaded

La credibilidad publicitaria en la nueva esfera mediática: los universitarios y los medios

Advertising credibility in the new media sphere: university students and the media 
Arco iris en medios brasileños: percepciones sociales sobre una campaña publicitaria LGBTQIA+

Rainbow in Brazilian media: social perceptions of an LGBTQIA+ advertising campaign

Leonardo Ferreira Batista, José Geraldo de Araújo Ramalho Filho

224-239

Rituales y comunicación política: la toma de posesión de los presidentes autonómicos españoles

Rituals and political communication: the Spanish autonomous Presidents inauguration

Ricardo Domínguez García

240-258

RESEÑAS REVIEWS

Una mirada diferente para hacer periodismo

A different look for making journalism

Guillermo Paredes-Otero

259-261

Periodismo y tecnología: una simbiosis continua

Journalism and technology: a continuous symbiosis

Luisa Graciela Aramburú Moncada

262-265

Una propuesta metodológica para impartir la asignatura de redacción periodística

Journalistic writing course: a methodological approach

Noelia Ruiz-Alba

266-268 


\title{
Una propuesta metodológica para impartir la asignatura de redacción periodística
}

\author{
Journalistic writing course: a methodological approach
}

\section{Pastora Moreno Espinosa}

Aportaciones metodológicas para la Redacción Periodística

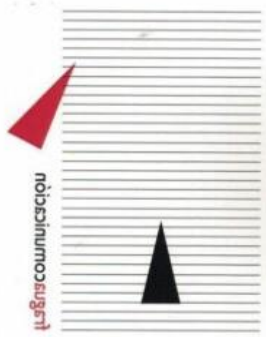

EDIropial
Aportaciones metodológicas para la Redacción

Periodística

Pastora Moreno Espinosa

Editorial Fragua, Madrid, 2019

195 páginas

Reseña por Noelia Ruiz-Alba

'Aportaciones metodológicas para la Redacción Periodística' está escrito por la primera mujer catedrática de periodismo en Andalucía, Pastora Moreno Espinosa, quien además de doctora en Comunicación lo es en Filología. Es profesora de Redacción Periodística desde el año 1992.

Este libro está estructurado en tres grandes bloques: Programa docente de redacción periodística (radio y televisión), Planteamientos docentes y Planteamientos investigadores. Como detalle, esta obra recomienda un cuerpo de trabajos complementarios para que los investigadores puedan ampliar el contenido expuesto en estas páginas. Además, la profesora Moreno recoge en el anexo los planes de estudio de Periodismo, Comunicación Audiovisual y Publicidad de la que es su casa: la Facultad de Comunicación de Sevilla.

En el primer apartado, Programa docente de Redacción periodística (radio y televisión), se explica que esta asignatura es obligatoria en el tercer curso de periodismo en la Facultad en la profesora ejerce la docencia. Tiene carácter cuatrimestral y persigue como objetivo principal "el Estudio Teórico Práctico del Mensaje y los Géneros Periodísticos y Redacción de los mismos en Radio y Televisión" así como el uso de "las nuevas tecnologías como herramientas de trabajo y conocimiento". Igualmente, tiene 
como finalidad que los estudiantes se formen sobre las distintas formas de redacción en los medios audiovisuales y analizar desde una perspectiva comparada los diferentes medios de comunicación. El programa se articula en dos bloques, uno dedicado a la radio y otro a la televisión.

Además, se advierte de que al alumnado que cursa esta materia se le presuponen unos conocimientos sobre la terminología propia de la redacción periodística, al igual que de las distintas formas del discurso. La autora explica que el mensaje periodístico tiene su manifestación específica los géneros periodísticos y que, durante mucho tiempo, se ha considerado que la construcción de la realidad se produce "desde ellos, con ellos y gracias a ellos". Esta afirmación nos recuerda al dicho en la profesión (siempre en tono crítico) "que la realidad no te estropee un buen titular".

Este libro recoge también las competencias de la asignatura y la metodología, epígrafe donde detalla el cronograma de esta formación que abarca un total de 60 horas lectivas, repartidas en 15 semanas. Se incluye un cronograma con las temáticas abordadas en clase. Este apartado puede resultar de interés para los investigadores que se inicien como docentes en esta asignatura, pues da buena cuenta de cómo se ha organizado la docencia de la profesora Pastora Moreno.

Como particularidades, la docente ofrece la posibilidad de realizar un trabajo con el que los pupilos tengan un primer contacto con la investigación académica, a la vez que imparte clases prácticas con el fin de estimular al futuro periodista a que salga a la calle "busque acontecimientos y los redacte". De este modo, sus estudiantes se convierten por vez primera en periodistas. En cuanto a criterios de evaluación, evita que la calificación dependa de una prueba final y persigue que el alumnado asista regularmente a las aulas. También añade el programa detallado de la asignatura así como una reseña bibliográfica y de recursos.

El segundo apartado, referido a los Planteamientos docentes, hace un repaso por la historia de la Facultad de Comunicación de Sevilla Facultad de Ciencias de la Información, como se llamaba otrora. Resulta llamativa la nómina de filólogos que impartían clases en la Facultad y que se adoptara el Plan de Estudios de la Universidad Autónoma de Barcelona, aspectos desconocidos para quienes que hemos estudiado en esas aulas recientemente.

Igualmente, esta obra hace unas precisiones conceptuales en torno a la redacción periodística, poniendo en valor que el redactor tiene como principal tarea "valorar las noticias" y abandona viejas definiciones en las que el redactor se limita a la "tarea de poner por escrito". Estas páginas ofrecen un recorrido por los antecedes, desde las Escuelas de Periodismo, hasta las Facultades Universitarias y hace una firme defensa de que la redacción periodística tiene lugar como ciencia autónoma dentro de las Ciencias de la Comunicación. También la considera como una ciencia interdisciplinar o como "ciencia puente" por sus conexiones con otras disciplinas.

Partiendo de la función social y el servicio público que son o deberían ser los medios de comunicación, la autora arroja luz sobre verdades a menudo silenciadas en la profesión. En esta dirección, expone que a veces los redactores deben sacrificar algunas noticias para mantener unas buenas y productivas relaciones con las fuentes. No dar cuenta de esta realidad sería ocultar el panorama actual a los futuros periodistas. Si bien el primer capítulo lo hemos recomendado para docentes que impartan por 
primera vez esta asignatura, este segundo es interesante para los propios alumnos ya que en él se detallan conceptos y se hace un recorrido por la historia de la redacción periodística.

La profesora detalla tres tipos de relaciones posibles con las fuentes: total independencia, cooperación entre fuente y periodista y la fuente que prácticamente hace la noticia. El tercer tipo de relación, desafortunadamente más común de lo que se desearía, va contra la misma definición que la autora hace de los medios de comunicación: función social y servicio público.

El último de los tres grandes apartados reconoce que todavía son pocos los manuales que ofrecen pautas para la comunicación periodística eficaz en la red. Reconoce que para el periodismo digital es de gran importancia la interacción y señala como tres características del lenguaje de este "nuevo" periodismo a la hipertextualidad, la multimedialidad y la interactividad.

Este libro, valiente por su contenido, critica la inversión que recibe la comunicación en España. Incluye la propuesta de un proyecto 'EgaudimMediaLab', que tiene como objetivo estudiar los periódicos digitales para comprobar si contienen los mismos géneros tradicionales y, como meta más ambiciosa, cómo crear una Cátedra de Redacción y MediaLab.

En definitiva, un libro útil, sencillo y fácil de leer que puede resultar de interés para docentes e investigadores que impartan por primera vez la asignatura de redacción periodística porque detalla la metodología empleada por la docente así como para estudiantes por su disertaciones conceptuales en torno a la redacción periodística. 